\title{
ELECCIONES DE 17 DE OCTUBRE DE 1993 AL PARLAMENTO GALLEGO
}

\author{
JUAN MANUEL GOIG MARTÍNEZ \\ Profesor Asociado del Departamento de Derecho Contitucional. \\ UNED
}




\section{SUMARIO}

I. Introducción.-II. La campaña electoral.-III. Resultados electoRALES.-Participación.-Resultados generales.-Resultados por circunscripciones electorales.-Resultados por Partidos Politicos y Coaliciones.-IV. Conclusıones. 


\title{
ELECCIONES DE 17 DE OCTUBRE DE 1993 AL PARLAMENTO GALLEGO
}

\author{
POR \\ JUAN MANUEL GOIG MARTINEZ \\ Profesor Asociado del Departamento de Derecho Constitucional. UNED
}

\section{INTRODUCCIÓN}

Las cuartas elecciones a la autonomía gallega dieron comienzo en la madrugada del día 2 de octubre de 1993, fecha de inicio de la campaña electoral tendente a determinar la fuerza de las expectativas electorales que tendrán su resultado el día 17 de octubre.

En total son trece los partidos y coaliciones que aspiran a ocupar los 75 escaños del Parlamento de Galicia en cuatro circunscripciones, de los que corresponden 24 a La Coruña, 15 a Lugo, 15 a Orense y 21 a Pontevedra, siendo el censo de 2.178.995, y los aspirantes a ocupar uno de los escaños 984 .

El número de mesas electorales que se constituirán será de 3.513 , con 10.539 miembros, de un total de 21.266 personas movilizadas' para el proceso electoral entre representantes de las distintas Administraciones y los 4.000 efectivos de las Fuerzas y Cuerpos de Seguridad del Estado que garantizarán la normalidad de la jornada.

El proceso electoral correrá a cargo de la Administración autonómica, correspondiendo a la Administración Central la garantía del orden y el suministro de la infraestructura material para el desarrollo de las votaciones.

1 Diario 16, 17 de octubre de 1993. M. A. Rodríguez. 


\section{LA CAMPAÑA ELECTORAL}

La campaña electoral, de 15 días de duración, comienza en medio de una cierta psicosis y una guerra de cifras sobre los resultados que arrojarán las urnas el próximo dia 17 de octubre. Aunque son 13 los partidos y coaliciones en contienda y 984 los aspirantes a obtener el acta de Diputado, las estimaciones atribuyen a sólo 4 de ellos posibilidades de obtener alguno de los escaños en liza. La última encuesta realizada antes del inicio de la campana pronostica la ratificación de la mayoría absoluta del PP y la derrota electoral para el PSOE — partidos que han polarizado la atención-, que puede perder varios escaños. Todos los líderes de la oposición coinciden en señalar el dia 17 como el principio de la "última fase de Manuel Fraga" "2, que desde que desembarcó en Galicia ha obtenido la victoria en todas sus comparecencias electorales.

Las elecciones gallegas del día 17, que abren la serie de citas con las urnas que jalonarán la vida pública española hasta el final -teóricode esta legislatura nacional (consultas europeas, andaluzas y vascas en 1994; las elecciones locales y autonómicas de 1995, y los comicios catalanes de 1996), tienen lugar a escasa diferencia en el tiempo de las elecciones legislativas de 6 de junio de 1993, elecciones generales que se convierten en el fantasma de la campaña electoral, fruto de las cifras de votos y a los resultados.

En las recientes elecciones generales se ha producido un fuerte retroceso del partido en el Gobierno central (PSOE), que ve reducidos a 159 sus Diputados y a 117 sus representantes en el Senado, frente. los 175 y 129 que obtuvo en 1989, respectivamente, y un ascenso del principal partido opositor (PP), que obtiene 141 Diputados -en 1989 había obtenido 107-y 107 Senadores - frente a los 91 con que contaba en la anterior legislatura- y que triunfa en todas las provincias gallegas, salvo Orense, donde a pesar de haber obtenido mayor número de votos, logra igual número de escaños que el PSOE ${ }^{3}$.

Curiosamente, los partidos nacionalistas, a pesar de que en las elecciones del 6 de junio se elegía al Parlamento nacional, ofrecen una equiparación de su representación frente a las anteriores legislativas.

Durante los 15 días de duración de la campaña, las 13 formaciones organizaron un promedio de 30 actos electorales diarios en el total de los

2 "Todos contra Fraga", Antón Reguera, El Mundo, 4 de octubre de 1993.

3 Datos del "B.O.E." n. ${ }^{\circ}$ 169, de 16 de julio de 1993. 
313 municipios de que consta la Comunidad gallega, con un gasto general que osciló en torno a los seiscientos millones de pesetas ${ }^{4}$. La reducción de los gastos electorales por la Ley Electoral ${ }^{5}$ y la diseminación de la población gallega ha supuesto un enorme esfuerzo por parte de las fuerzas políticas de realizar una amplia campaña, de carácter "puerta a puerta", siendo Manuel Fraga el que en mayor número de actos ha participado ${ }^{6}$, y un amplio sistema de ajuste de los presupuestos electorales, aunque alguna formación se haya sobrepasado en los limites ${ }^{7}$.

La campaña, que estuvo marcada en todo momento por los insultos personales entre los principales candidatos en contienda y por las continuas acusaciones y defensas respecto al pasado franquista del candidato del PP, tuvo como fondo la situación de crisis que afecta al Estado $y$, ciñéndonos al ámbito territorial de la campaña, a la crítica situación de la economía gallega ${ }^{8}$, a lo que hay que unir el polémico tema de la cesión, por parte del Estado, del 15\% del IRPF a las Comunidades Autónomas, a la que Galicia se opuso en el Consejo de Política Fiscal alegando que el reparto perjudica a la Comunidad Gallega ${ }^{9}$.

La juventud se convierte en un importante argumento electoral para las fuerzas políticas ${ }^{10}$, que intentan atraerse el mayor número de nuevos votantes, $y$, pese a su escasa significación cuantitativa (el $4 \%$ del electorado), el llamado "voto emigrante" " es igualmente codiciado por los partidos en contienda.

A pesar de los enfrentamientos y acusaciones en temas de comunicación ${ }^{12}$, uno de los alicientes de la campaña fue el esperado debate que mantuvieron los dos principales candidatos en Antena 3 Televisión,

4 A estas cifras hay que añadir el desembolso de la Xunta, superior a 860 millones. EI Mundo, 13 de octubre de 1993.

5 Se limita a 198.000 .000 ptas. lo que puede gastar el partido mayoritario.

- El Mundo, 12 de octubre de 1993, Francisco Pedroso.

1993.

7 Concretamente el Partido Popular. La Voz de Galicia, 12 de octubre de

8 "Todos contra Fraga", El Mundo, 4 de octubre de 1993, A. Reguero.

9 El País, 9 de octubre de 1993, Javier Rivas.

10 De los nuevos votantes en las elecciones generales de 6 de junio, el PP obtiene 545.000, frente a los 471.000 del PSOE. El Mundo, 13 de junio de 1993, estudio realizado por C. Malo, J. M. Elias y C. Prieto, según estudio de Sigma 2.

"En La Coruña tienen derecho a voto 31.100 emigrantes; en Pontevedra, 24.435; en Orense, 17.125, y en Lugo, 9.114. El Mundo, 15 de octubre de 1993.

12 Estos problemas hacen referencia al "vídeo" de Manuel Fraga y a la presunta censura de un mitin de Felipe González en la Televisión Gallega, El Pais, 13 de octubre de 1933. 
primera vez que sucede en una elecciones autonómicas, $v$ que resultó con un empate entre los lideres ${ }^{13}$.

Tras los resultados de las encuestas que los principales medios de comunicación hacen, previo al 17 de octubre, y que vienen a confirmar la ascensión del PP y del BNG, y la pérdida de votos del PSOE, los líderes políticos de estas formaciones se esforzarán, bien por ponderar estos resultados, bien por pedir al electorado que hagan caso omiso a los mismos (*).

Partidos con opción a escaño y sus líderes políticos

\begin{tabular}{ll}
\hline PP & Manuel Fraga Iribarne \\
BNOE-PSG & Antolín Sánchez Presedo \\
UG-EU & Cosé Manuel Beirás \\
CG & Cémilo Nogueira Román
\end{tabular}

Uno de los aspectos más importantes a tener en cuenta en la campaña es el apoyo que todos los líderes piden a la participación electoral, ya que Galicia es la Comunidad Autónoma que ha registrado una mayor abstención, sobre todo en las elecciones autonómicas, tal vez fruto de la desconfianza y la falta de consolidación de la autonomía, y debido a las características geopolíticas y de disgregación de la población.

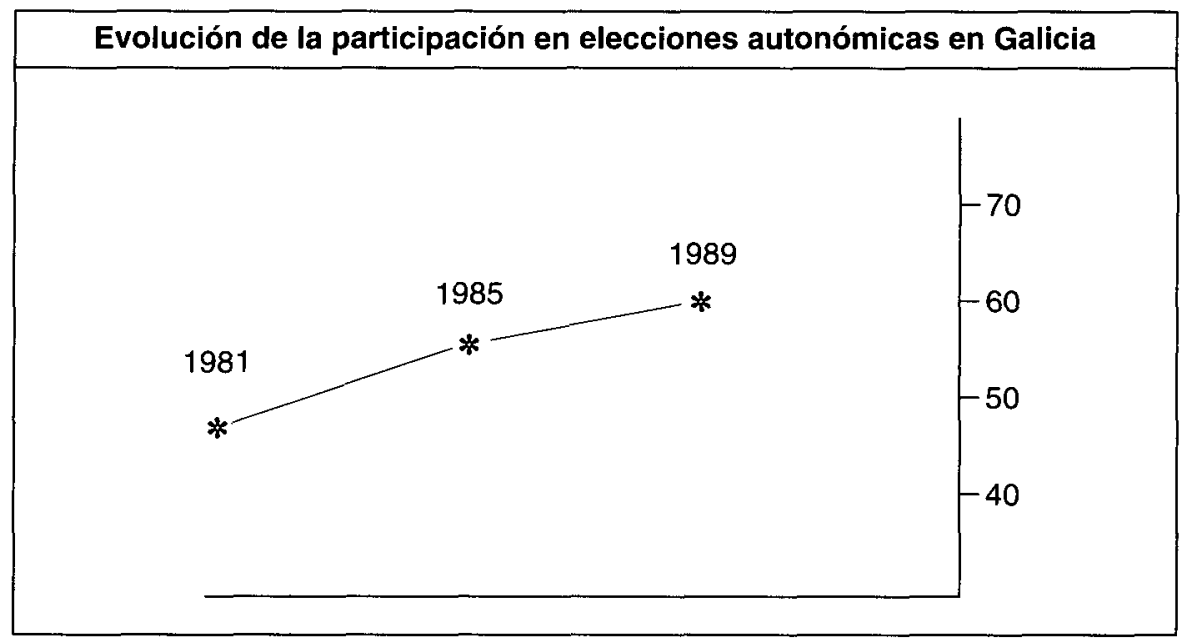

13 Diario 16, 6 de octubre de 1993. 


\section{Sondeo de Demoscopia}

(El Pais, 10 de octubre de 1993)

\begin{tabular}{ccccc}
\hline Escaños & Partidos politicos & Escaños 1993 & Escaños 1989 & Participación \\
\hline & PP & 43 & 38 & \\
\cline { 2 - 4 } 75 & PSOE & $19-21$ & 28 & Participación \\
\cline { 2 - 4 } & EVNG (*) & $0-2$ & 2 & $66 \%$ \\
\cline { 2 - 4 } & BNG & 11 & 5 & Abstención \\
& CG & - & - & $34 \%$ \\
& Otros & - & - & \\
\hline
\end{tabular}

Sondeo de Sigma Dos

(El Mundo, 10 de octubre de 1993)

\begin{tabular}{ccccc}
\hline Escaños & Partidos politicos & Escaños 1993 & Escaños 1989 & Participación \\
\hline \multirow{7}{*}{75} & PP & 39 & 38 & (5) \\
\cline { 2 - 4 } & PSOE & 24 & 28 & \\
\cline { 2 - 4 } & EVNG (*) & 1 & 2 \\
\hline & BNG & 11 & 5 \\
\hline & CG & - & - \\
\hline
\end{tabular}

Sondeo de ICP/Research

(Diario 16, 16 de octubre de 1993)

\begin{tabular}{ccccc}
\hline & PP & $43-39$ & 38 & \\
\cline { 2 - 4 } 75 & PSOE & $24-22$ & 28 & Participación \\
\cline { 2 - 3 } & EVNG $\left(^{*}\right)$ & $1-2$ & 2 & $61,9 \%$ \\
\cline { 2 - 4 } & BNG & $10-9$ & 5 & Abstención \\
& CG & 0 & 2 & $38,1 \%$ \\
\cline { 2 - 3 } & Otros & - & - & \\
\hline
\end{tabular}

(*) Antes EG

(5) No ofrece datos de participación. 
El último aspecto importante a destacar es el carácter central de los principales partidos contendientes, frente al carácter nacionalista de los partidos y coaliciones ganadores de las elecciones autonómicas catalana y vasca, y el escaso, aunque no por ello menos importante, papel de los partidos nacionalistas gallegos que, a pesar de aspirar a convertirse en importante fuerza opositora, no aspiran a obtener la mayoría suficiente para formar gobierno monocolor.

Las pretensiones de las distintas formaciones políticas que se han puesto de manifiesto en la campaña se pueden resumir de la siguiente forma:

Partido Popular (PP)

Fraga ha pedido el apoyo de los gallegos para dar un nuevo impulso "hacia delante a Galicia" en los próximos 4 años. Las pretensiones del candidato popular a la Xunta consisten en revalidar la mayoria absoluta nuevamente, puesto que «son necesarios dos períodos para poder culminar el trabajo realizado" ${ }^{14}$, y para evitar el posible pacto a tres bandas por parte de los partidos opositores que subyace en la mente de los candidatos ${ }^{15}$. La idea fundamental de la campaña se centra en la consecución de que Galicia avance decididamente a la modernidad sin complejos y dispuesta a asumir los retos ${ }^{16}$, para lo que se combate por todos los medios la abstención y se solicita un aumento de escaños, movidos por la euforia de las encuestas y de los resultados electorales de las elecciones legislativas del 6 de junio, en las que el PP experimentó un gran avance electoral.

Para la consecución de estos fines, el PP ofrece su imagen de moderación y las consecuciones de la Xunta en infraestructura, a lo que se une su intento de rentabilizar al máximo el Xacobeo 93. Otro de los mayores intentos del candidato consistiría en lograr la división del voto de los progresistas, en detrimento de una concentración de apoyo al PSOE.

14 Diario 16, 16 de octubre de 1993, Carlota Corredera.

15 Diario 16, 12 de octubre de 1993: "Aquí no vale empate, hay que arrasar por goleada", dice el lider conservador.

16 Manuel Fraga, "Una Galicia de todos para todos", El Mundo, 15 de octubre de 1993. 
Partido Socialista (PSG-PSOE)

Busca evitar la mayoría absoluta del PP, para la realización de un posible pacto de Gobierno con BNG y EU-UG, aunque de producirse éste, el PSOE es consciente de su atadura a las reivindicaciones nacionalistas $^{17}$. No obstante, podemos afirmar que arrastra tres aspectos negativos: la forma en que el PSOE llegó a formar gobierno en Galicia entre 1987 y 1989 (mediante el voto de 5 tránsfugas que provenían de las listas conservadoras); la crisis económica en la que se encuentra el Estado, y la profunda crisis interna (guerristas-renovadores) que está padeciendo el partido y que se ha manifestado en Galicia con la presentación de un candidato perteneciente al "sector renovador".

Para los socialistas, aparte del test específicamente gallego, estas elecciones suponen un test sobre el estado actual del socialismo en España ${ }^{18}$. Por muchas correcciones que haya que efectuar a la extrapolación de los resultados gallegos, un descenso significativo del voto constituiría un sintoma de que el deterioro del PSOE se ha acentuado. Las acusaciones al PP de discurso antiautonomista ${ }^{19}$ y los ataques al partido opositor frente a la crítica situación económica, son bazas a jugar en la campaña del PSOE.

\section{Bloque Nacionalista Gallego (BNG)}

Nunca una fuerza política gallega que propugna la ruptura del actual modelo de Estado ha obtenido tanto respaldo social. EI BNG aspira a culminar el 17 de octubre una trayectoria que va desde el testimonialismo a la posibilidad, según las encuestas, de obtener el apoyo del $15 \%$ del electorado.

Xosé Manuel Beirás, definido enemigo del candidato del PP ${ }^{20}$, aspira a arrebatar al PP los votos suficientes para destruir su mayoría absoluta y gobernar con el PSOE. EI núcleo originario de este frente nacionalista es Unión do Pobo Gallego (UPG), partido marxista-leninista, que

17 El Mundo, 4 de octubre de 1993, "Todos contra Fraga", A. Regueira.

18 Editorial de Diario 16,17 de octubre de 1993.

19 El Mundo, 11 de octubre de 1993.

20 Xosé Manuel Beirás: "Sólo hablo de mis enemigos, o sea, de Fraga", Diario 16,13 de octubre de 1993. 
con los años ha ensanchado su base con la incorporación de grupos socialdemócratas y socialistas. La lucha por la restauración de las reglas del juego democrático en la política gallega, tanto en las instituciones como en la sociedad, y el enfrentamiento a la llamada al "voto útil" del PSOE, son los puntos que centran la campaña de esta formación, que podría ser la gran beneficiaria del revés electoral del PSOE que auguran las encuestas.

\section{Unidade Galega-Esquerda Unida (UG-EU)}

Es el tercer partido que se autoproclama de izquierdas ${ }^{21}$ que compite electoralmente para arrebatar al PP la mayoría absoluta. Parte de la idea de que es necesario remediar la ausencia de Galicia como nacionalidad en la política estatal y europea ${ }^{22}$. Defiende la concepción de que Galicia tiene su propia personalidad como nación europea y aspira a hacer del Estado español una confederación o federación amplia donde incluso las competencias ejecutivas que conserve el Estado sean ejercidas por las instituciones políticas gallegas.

Su campaña se movilizó plenamente cuando algunas encuestas electorales vaticinaron la pérdida de sus dos Diputados autonómicos. Su adscripción al nacionalismo democrático de izquierdas determina su apoyo al PSOE en el caso de que el PP no consiga la mayoría absoluta, pero condicionado a una modificación del PSOE en su actuación central y comunitaria.

\section{Coalición Galega (CG)}

Con un programa denso y moderno comparece ante el electorado este partido que tuvo 11 Diputados en Galicia y uno en Madrid, que en las autonómicas de 1989 obtuvo sólo 2 Diputados y al que las encuestas electorales auguran su desaparición del Parlamento Gallego.

Se presenta con la idea de asentar un nacionalismo moderado al margen del "inmovilismo de la derecha y de la izquierda obsoleta» ${ }^{23}, y$

\footnotetext{
21 El País, 15 de octubre de 1993, José Hermida.

22 El Mundo, 13 de octubre de 1993, Francisco Fredoso.

23 El Mundo, 13 de octubre de 1993, Francisco Fredoso.
} 
aspira a la existencia de una alternativa frente al centralismo. Ha recibido el apoyo del PNV (Partido Nacionalista Vasco), por entender que esta formación «representa al nacionalismo histórico gallego, al centro político y a la defensa de los derechos humanos y la justicia social" ${ }^{24}$.

\section{RESULTADOS ELECTORALES}

Sin apenas incidentes, si excluimos los problemas de lluvia ocasionados en algunas circunscripciones electorales, y algunos errores en el censo electoral, se desarrolló la jornada electoral, en la que los gallegos ejercieron de manera clara sus derechos democráticos.

\section{Participación}

Con una participación del $65,62 \%{ }^{25}$ se acaba con la excepcionalidad de un abstencionismo muy superior al de otras Comunidades Autónomas ${ }^{26}$, que marcó el inicio del proceso autonómico $y$ cuyo dato más alarmante lo constituya el Referéndum de ratificación del Estatuto, en diciembre de 1980 , en el que la participación no supera el $30 \%$.

La importancia de esta alta participación se aprecia más si se tiene en cuenta que en las legislativas del 6 de junio - $y$ en Galicia las elecciones legislativas han llevado a muchos más ciudadanos a las urnas que los comicios autonómicos- la participación en toda Galicia llega a un 71\%.

Tal vez los problemas surgidos en la política nacional -el problema de la gobernabilidad- $y$ los acuerdos entre fuerzas heterogéneas hayan movido al pueblo gallego a reducir su abstención en estos comicios, que han estado marcados por una posible opción entre la continuidad y la inestabilidad y el desasosiego ${ }^{27}$. Pero sea cual sea la causa, es de aplaudir el deseo del pueblo gallego por el reforzamiento en la consolidación de su autonomía.

24 El Mundo, 16 de octubre de 1993.

25 Datos oficiales ofrecidos por la Consejería de Presidencia de la Xunta.

${ }^{26}$ La abstención en las recientes elecciones al Parlamento Catalán llegó al $45,2 \%$. M. Carmen Fernández-Miranda, RDP, n. 938.

27 El País, Opinión, "El gran triunfo de Fraga», 18 de octubre de 1993. 


\section{Datos generales de participación}

Censo

N.․ Mesas Electorales

N.. Votantes

Escaños
2.193.482

3.513

1.442 .775

75

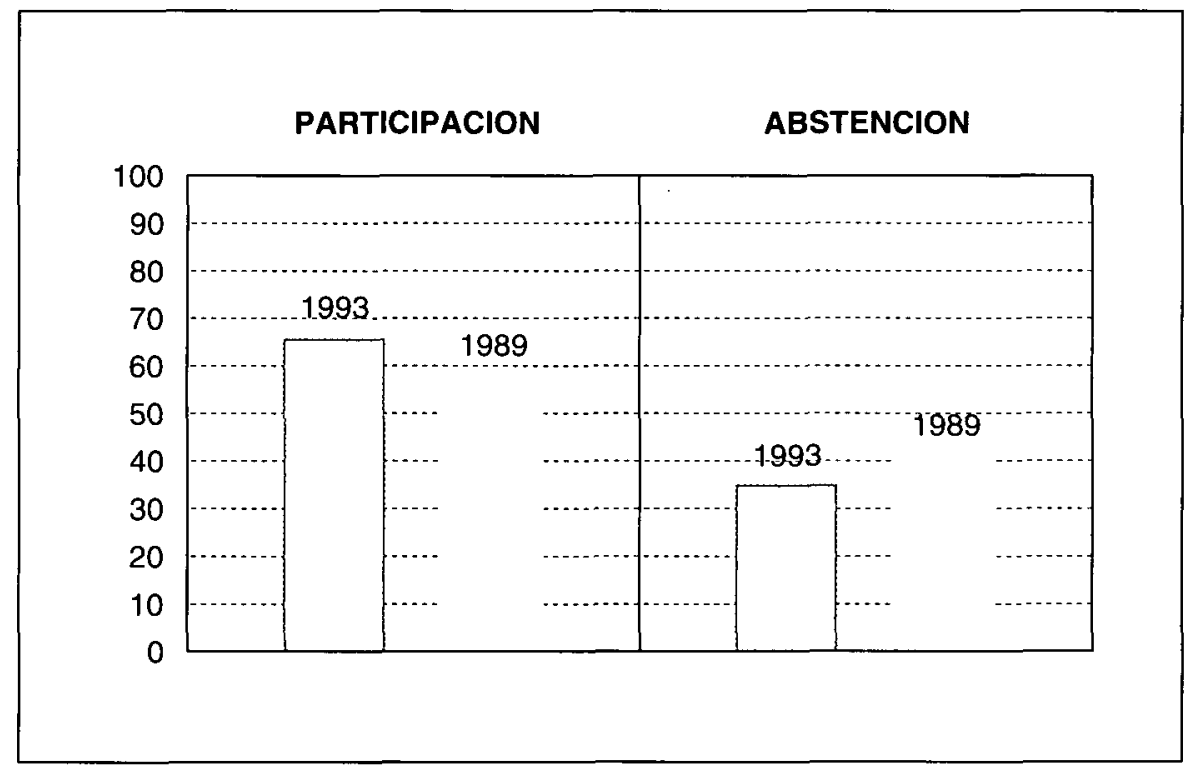




\section{Resultados generales}

\begin{tabular}{|c|c|c|c|}
\hline \multicolumn{4}{|c|}{1993} \\
\hline Candidaturas & Votos & $\%$ & Escaños \\
\hline PP & 749.961 & 52,21 & 43 \\
\hline PSG-PSOE & 337.048 & 23,47 & 19 \\
\hline BNG & 268.248 & 18,68 & 13 \\
\hline EV-VG & 44.614 & 3,11 & - \\
\hline CG & 6.017 & 0,42 & - \\
\hline ARM & 5.245 & 0,37 & - \\
\hline 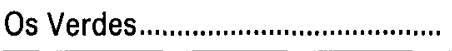 & 4.381 & 0,31 & - \\
\hline AG & 3.080 & 0,21 & - \\
\hline LE & 2.733 & 0,19 & - \\
\hline PCH & 2.750 & 0,18 & - \\
\hline APU & 1.474 & 0,10 & $一$ \\
\hline NPS & 498 & 0,03 & - \\
\hline \multicolumn{4}{|c|}{1989} \\
\hline Candidaturas & Votos & $\%$ & Escaños \\
\hline 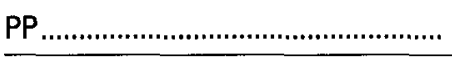 & 582.157 & 44,08 & 38 \\
\hline PSG-PSOE & 429.571 & 32,53 & 28 \\
\hline BNG & 105.698 & 8,00 & 5 \\
\hline EV-VG & 70.117 & 5,31 & 2 \\
\hline 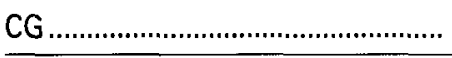 & 48.297 & 3,66 & 2 \\
\hline ARM & 7.084 & 0,54 & - \\
\hline 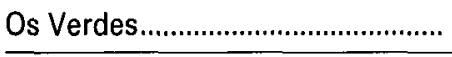 & 3.150 & 0,24 & - \\
\hline AG & - & - & - \\
\hline 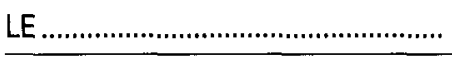 & - & - & - \\
\hline PCH & 757 & 0,06 & - \\
\hline APU & - & - & - \\
\hline NPS & - & - & - \\
\hline
\end{tabular}


Resultados generales (continuación)

\section{Diferencias entre 1993 - 1989}

\begin{tabular}{|c|c|c|c|}
\hline Candidaturas & Votos & $\%$ & Escaños \\
\hline 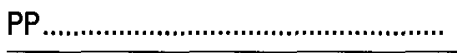 & +167.804 & $+8,13$ & +5 \\
\hline PSG-PSOE & -92.523 & $-9,06$ & -9 \\
\hline 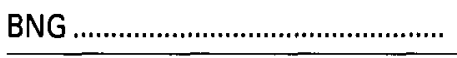 & +162.550 & $+10,68$ & +8 \\
\hline EV-VG & -25.503 & $-2,20$ & -2 \\
\hline CG & -42.280 & $-3,24$ & -2 \\
\hline ARM & -1.739 & $-0,17$ & - \\
\hline 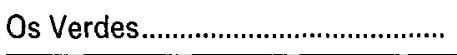 & +1.231 & $+0,07$ & - \\
\hline 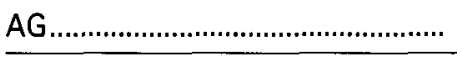 & $*+3.080$ & $*+0,21$ & - \\
\hline LE & ${ }^{*}+2.733$ & $*+0,19$ & - \\
\hline $\mathrm{PCH}$ & +1.813 & $+0,12$ & - \\
\hline APU & ${ }^{*}+1.474$ & $*+0,10$ & - \\
\hline NPS & ${ }^{*}+498$ & ${ }^{*}+0,03$ & - \\
\hline
\end{tabular}




\section{Resultados por Circunscripciones Electorales}

\section{Circunscripción de La Coruña}

Censo

N.․ Votantes

N.- Mesas Electorales

Escaños

Participación

Abstención
890.273

566.541

1.330

24

$63,64 \%$

$36,36 \%$

Relación con 1989

\begin{tabular}{|c|c|c|c|c|c|}
\hline Candidaturas & N.․ votos & $\%$ & Escaños & $\%$ & Escaños \\
\hline PP .......................... & 267.118 & 48,97 & 13 & $+8,05$ & +2 \\
\hline PSG-PSOE............. & 137.925 & 24,46 & 6 & $-10,54$ & -4 \\
\hline 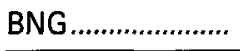 & 112.230 & 19,90 & 5 & $+10,54$ & +3 \\
\hline EU-UG ...................... & 21.156 & 3,75 & - & $-2,36$ & -1 \\
\hline
\end{tabular}

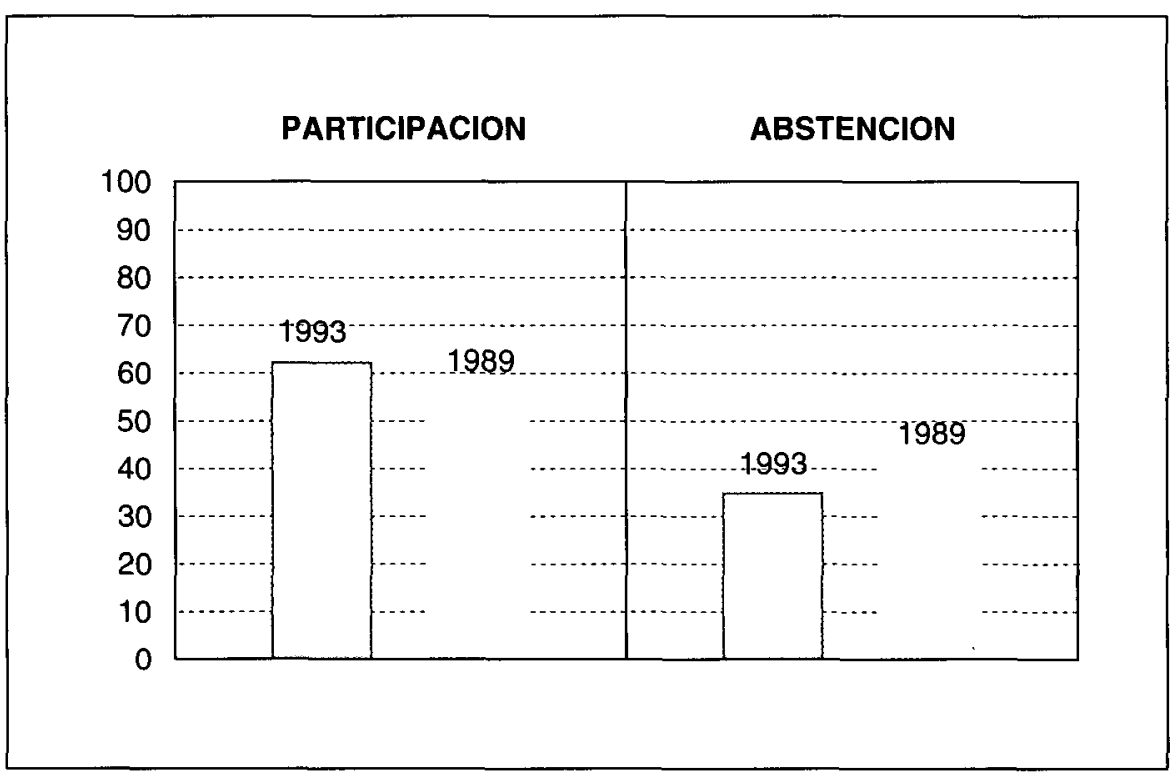


Circunscripción de Lugo

Censo

315.743

N. Votantes

215.282

NN. Mesas Electorales

549

Escaños

15

Participación

$68,18 \%$

Abstención

$31,82 \%$

Relación con 1989

\begin{tabular}{lrrccc}
\hline Candidaturas & N.. votos & $\%$ & Escaños & $\%$ & Escaños \\
\hline PP ..................... & 121.337 & 56,67 & 9 & $+8,66$ & +1 \\
\hline PSG-PSOE.......... & 49.075 & 22,92 & 4 & $-9,84$ & -1 \\
\hline BNG .................... & 35.750 & 16,70 & 2 & $+10,05$ & +1 \\
\hline CG ...................... & 920 & 0,43 & - & $-6,82$ & -1 \\
\hline
\end{tabular}

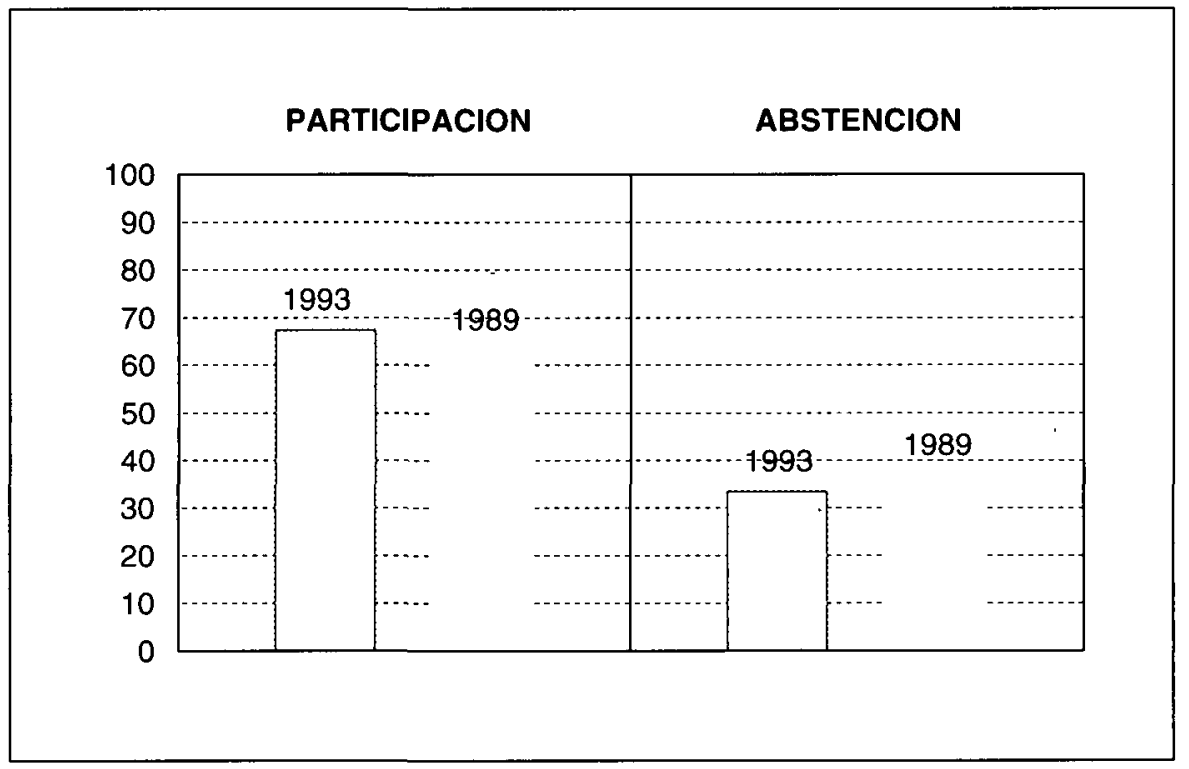


Circunscripción de Orense

Censo.

N.․ Votantes

N.․ Mesas Electorales

Escaños

Participación

Abstención
293.575

196.065

547

15

$66,79 \%$

$33,21 \%$

Relación con 1989

\begin{tabular}{lrcccc}
\hline Candidaturas & N.. votos & $\%$ & Escaños & $\%$ & Escaños \\
\hline PP .................... & 106.688 & 54,63 & 9 & $+10,36$ & +1 \\
\hline PSG.PSOE.......... & 49.134 & 25,15 & 4 & $-7,38$ & -2 \\
\hline BNG .................... & 33.795 & 17,30 & 2 & $+11,88$ & +2 \\
\hline CG.................... & 986 & 0,50 & - & $-5,96$ & -1 \\
\hline
\end{tabular}

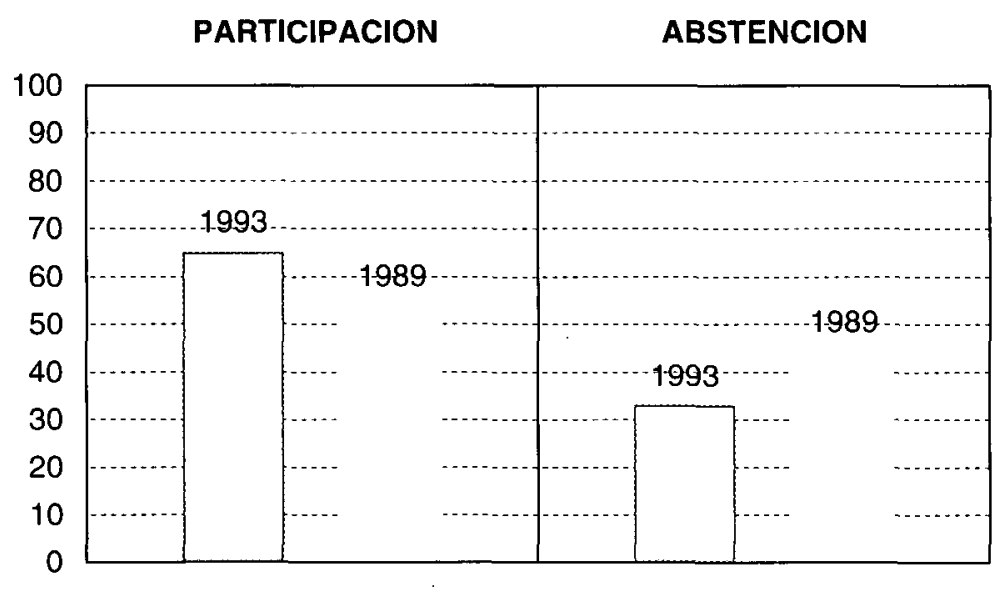


Circunscripción de Pontevedra

Censo

N.․ Votantes

N.. Mesas Electorales

Escaños

Participación

Abstención
693.891

464.887

1.087

21

$67,00 \%$

$33,00 \%$

Relación con 1989

\begin{tabular}{lccccc}
\hline Candidaturas & N.. votos & $\%$ & Escaños & $\%$ & Escaños \\
\hline PP ...................... & 245.818 & 53,08 & 12 & $+7,12$ & +1 \\
\hline PSG-PSOE ............ & 100.914 & 21,79 & 5 & $-8,30$ & -1 \\
\hline BNG ................... & 86.473 & 18,67 & 4 & $+10,51$ & +2 \\
\hline CG..................... & 18.224 & 3,94 & - & $-2,77$ & -1 \\
\hline
\end{tabular}

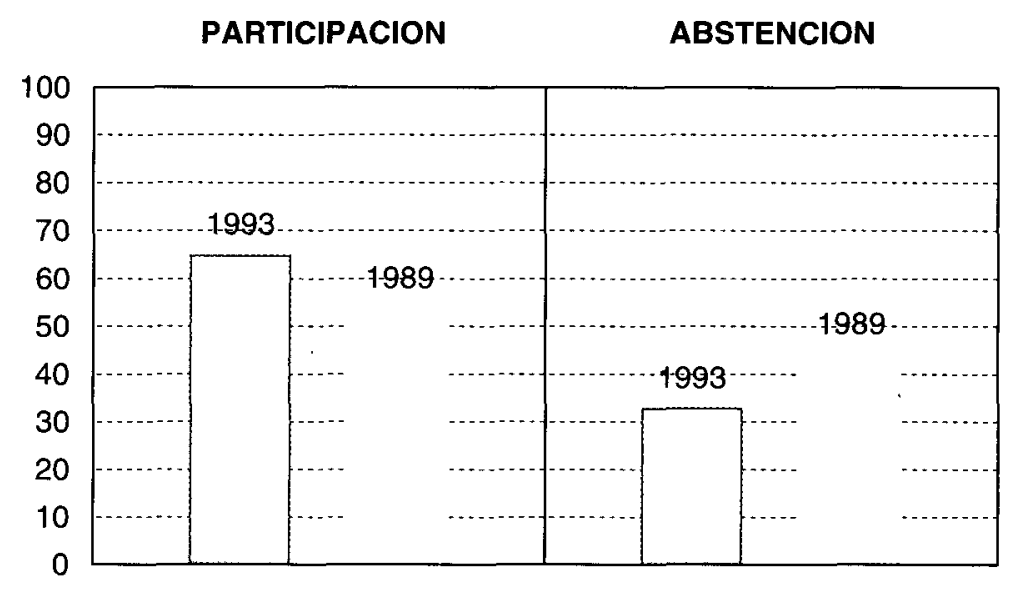




\section{Resultados por Partidos Políticos y Coaliciones}

\section{Partido Popular (PP)}

Las previsiones de las encuestas electorales son desbordadas por los resultados del PP, cuya mayoría absoluta se cimenta en más de la mitad de los votos depositados en las urnas, con un crecimiento del $44 \%$ al $52,33 \%$. El PP arrasa en 300 de los 313 Ayuntamientos ${ }^{28}$, obteniendo la victoria, por tanto, en el $92 \%$ de los mismos, e incluso ve aumentada su diferencia respecto al PSOE en La Coruña, donde la distancia entre ambas formaciones siempre ha sido la más estrecha. Además, Manuel Fraga consigue romper el récord electoral y techo histórico del líder popular nacional, José María Aznar.

Pero quizás el hecho más importante para el PP es que ve aumentada su victoria respecto a los comicios generales, lo que viene a confirmar el escepticismo del pueblo respecto a los resultados del 6 de junio.

\section{Partido Socialista (PSOE-PSG)}

Si existe un perdedor en estas elecciones es el PSOE, que sufre un enorme retroceso, prácticamente equivalente al ascenso del PP. En estos comicios los socialistas pierden casi ocho puntos en el cómputo de votos y nueve escaños, retroceso mayor que el experimentado en las elecciones al Parlamento Nacional.

La desconfianza del pueblo, la fuerte crisis económica y la división interna del partido, unido a la falta de carisma del líder socialista, pueden ser tenidas en cuenta como causas de esta derrota.

\section{Bloque Nacionalista Gallego (BNG)}

La formación liderada por Beirás ha sido la que ha experimentado un ascenso más notable. Gana 8 escaños - pasa de 5 a 13-, consigue un porcentaje de voto cercano al $19 \%(18,73 \%)$ y se consolida como la única

28 Diario 16, 19 de octubre de 1993, A. Alonso. 
fuerza nacionalista del Parlamento Gallego con serias aspiraciones para disputar a los socialistas el segundo lugar en el espacio político gallego.

Los resultados afianzan al Bloque como el tercer partido más importante de Galicia y le otorgan un papel predominante en esta nueva legislatura, hasta el punto de ensombrecer la incontestable victoria del $\mathrm{PP}^{29}$. Su líder ha conseguido agrupar en torno a sus listas prácticamente todo el voto nacionalista de comicios anteriores, fruto de lo cual ha sido la desaparición del arco parlamentario gallego de Coalición Gallega y la ruptura del bipartidismo en el Parlamento Gallego. Por otra parte, al tratarse de un nacionalismo de izquierdas, ha destruido las expectativas de Unidade Gallega, que ha quedado extinta ${ }^{30}$.

\section{CONCLUSIONES}

Los comicios se plantearon como una batalla entre un bloque conservador homogéneo, encabezado por un líder indiscutible en ese sector, y un bloque opositor heterogéneo y sin otro terreno compartido que el rechazo a Fraga.

\section{Evolución del voto autonómico en Galicia}

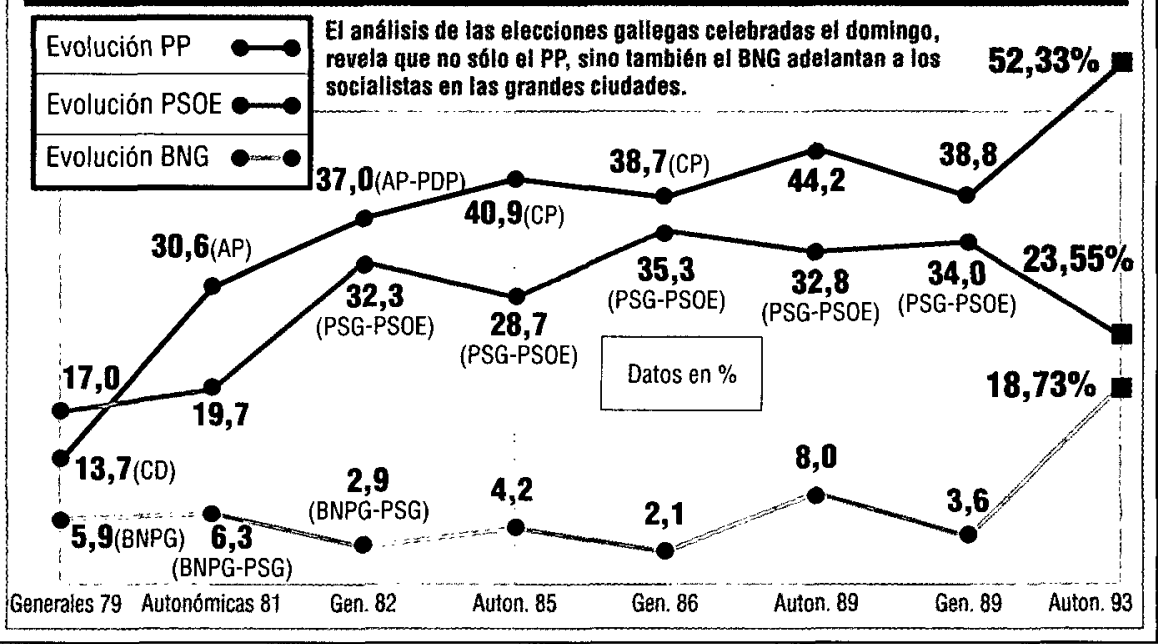


Si bien la comparación entre elecciones generales y autonómicas requiere extremar las precauciones, la cercanía, en este caso, y el carácter nacional de los principales partidos enfrentados en las elecciones generales, permite márgenes suficientes.

Mientras los votos populares en las elecciones autonómicas crecieron algo menos del $2 \%$ sobre las legislativas, el PSOE perdió casi el $40 \%, y$, por su parte, el BNG duplica sus resultados, toda vez que vemos desaparecer los escaños de CG y UG-EU.

Entre las causas de estos resultados se puede observar ${ }^{31}$, de un lado, que el voto útil depositado el 6 de junio en favor del PSOE, por temor a una posible victoria del PP, o bien se ha abstenido o ha optado por otra candidatura. De otro, un deseo de castigo al PSOE producido, entre otras causas, por los pactos de Gobierno con CiU y PNV, que pueden haber provocado un agravio en Galicia, por la crisis económica que todavía no ha tocado fondo o por los escasos resultados de los primeros 100 días de gobierno en lo que a impulso democrático se refiere.

31 "El escaparate gallego", Javier Pradera, El País, 24 de octubre de 1993. 